\title{
Stability and Sensitivity Analysis of the Fractional Order Dengue Model
}

\author{
Nur 'Izzati Hamdan'* and Adem Kilicman² \\ ${ }^{1}$ Department of Mathematics, Faculty of Science, Universiti Putra Malaysia \\ ${ }^{2}$ Institute for Mathematical Research (INSPEM), Universiti Putra Malaysia
}

\begin{abstract}
In this paper, a model for the dengue transmission is presented using the fractional order derivative in the sense of the Caputo derivative. The basic reproduction number denoted by $\mathrm{R}_{\mathrm{o}}$ is computed using the nextgeneration matrix approach. The local and global stability of the disease-free equilibrium is performed, and the existence of the positive endemic equilibrium is obtained for $R_{0}>1$. Further, sensitivity analysis is conducted to determine how changes in parameters affect the initial disease transmission of dengue.
\end{abstract}

Keywords: dengue, stability, sensitivity, epidemic, fractional

\section{INTRODUCTION}

Dengue is a fast-emerging pandemic-prone viral disease in many parts of the world, especially in the tropical and subtropical countries. The transmission process involved human and Aedes mosquitoes, primarily Aedes aegypti. The virus is transmitted to humans by the bites of an infected Aedes mosquito. There are four serologically different viruses, namely DEN I, II, III, and IV that can cause dengue disease (WHO, 2018).

Various mathematical models have been developed and analysed to understand the dynamics of dengue transmission. Most of the proposed models (Derouich et. al., 2003; Esteva \& Vargas, 1998; Esteva \& Vargas, 1999; Pinho et. al., 2010; Soewono \& Supriatna, 2001) is an extended model of susceptible-infected-recovered (SIR) model introduced by Kermack and McKendrick in 1927 (Kermack \& McKendrick, 1927). However, as the idea of fractional calculus being introduced, many researchers found that modelling infectious disease using the fractional order derivative is more realistic compared to the classical integer order derivative. Fractional order derivative provides a memory effect, where most of the biological systems have it.
Different dengue epidemic model (Diethelm, 2013; Pooseh et. al., 2011; Sardar et. al., 2014; Sardar et. al., 2015) have been proposed to study the dynamics of the dengue transmission using the fractional order derivative. But none of the models includes aquatic stages of the mosquito population. In the present work, we study the dengue epidemic model established in (Hamdan \& Kilicman, 2018; Hamdan \& Kilicman, 2019), but here, we consider all the dimension parameters to have a memory effect. Thus, parameters will be dependent on the order of the derivative, denoted by $\alpha$.

This paper is organized as follows: the formulation of the fractional order dengue epidemic model is briefly described in Section 2. In section 3, the stability analysis of the equilibrium points is presented. Local sensitivity analysis is performed in section 4 based on the normalized forward sensitivity index of the basic reproduction number, Ro. Using numerical computation, we simulate the importance of our results in section 5. Finally, the conclusion of our study is given in section 6 .

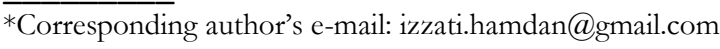


Table 1: Description of the model parameters.

\begin{tabular}{cl}
\hline Parameter & \multicolumn{1}{c}{ Description } \\
\hline $\mathrm{q}$ & $\begin{array}{l}\text { Proportion of eggs that } \\
\text { results in female mosquito }\end{array}$ \\
$\phi$ & Oviposition rate \\
$\sigma_{\mathrm{A}}$ & Transition rate from aquatic \\
& stage to adult \\
$\mu_{\mathrm{A}}$ & Per capita mortality rate of \\
& aquatic stage \\
$\mu_{\mathrm{m}}$ & Per capita mortality rate of \\
& mosquito \\
$\mu_{\mathrm{h}}$ & $\begin{array}{l}\text { Per capita mortality rate of } \\
\text { human }\end{array}$ \\
& The biting rate \\
$\beta_{\mathrm{m}}$ & Transmission probability \\
& from human to vector \\
$\beta_{\mathrm{h}}$ & Transmission probability \\
& from vector to human \\
$\gamma_{\mathrm{h}}$ & Recovery rate in the human \\
& population \\
$\mathrm{C}$ & Mosquito carrying capacity \\
\hline &
\end{tabular}

\section{MATHEMATICAL MODEL}

In this study, the Caputo derivative is used in fractionalize the integer order differential equation. The definition of the Caputo derivative is given as follows (Petras, 2011):

Definition 1 The Caputo derivative with order $\alpha$ of a function $f(t)$ is given by:

$$
{ }_{a}^{C} D_{t}^{\alpha} f(t)=\frac{1}{\Gamma(\mathrm{n}-\alpha)} \int_{a}^{t}(t-\tau)^{n-\alpha-1} f^{(n)}(\tau) d \tau(1) \text { where } \alpha \text { is }
$$

the order of the derivative with

$$
n-1<\alpha<n, n \in \mathbb{Z}^{+} .
$$

In the construction of the model, the total human population at time $\mathrm{t}$, denoted by $\mathrm{H}(\mathrm{t})$ is divided into three classes: $\mathrm{H}_{\mathrm{s}}$ (susceptible), $\mathrm{H}_{\mathrm{i}}$ (infected), $\mathrm{H}_{\mathrm{r}}$ (recovered), meanwhile, the total mosquito population $\mathrm{M}$ is divided into two classes: $M_{s}$ (susceptible), $M_{i}$ (infected). The aquatic phase of the mosquito in denoted by $A_{m}$, represents immature stage including egg phase, larva, and pupa. The basic model for the transmission dynamics of dengue in the integer order sense is given by the following deterministic system of nonlinear differential equations:

$$
\frac{d A_{m}}{d t}=q \phi\left(1-\frac{A_{m}}{C}\right) M-\left(\sigma_{A}+\mu_{A}\right) A_{m},
$$

$$
\begin{aligned}
\frac{d M_{s}}{d t} & =\sigma_{A} A_{m}-\frac{b \beta_{m}}{H} M_{s} H_{i}-\mu_{m} M_{s}, \\
\frac{d M_{i}}{d t} & =\frac{b \beta_{m}}{H} M_{s} H_{i}-\mu_{m} M_{i}, \\
\frac{d H_{s}}{d t} & =\mu_{h}\left(H-H_{s}\right)-\frac{b \beta_{h}}{H} H_{s} M_{i}, \\
\frac{d H_{i}}{d t} & =\frac{b \beta_{h}}{H} H_{s} M_{i}-\left(\gamma_{h}+\mu_{h}\right) H_{i}, \\
\frac{d H_{r}}{d t} & =\gamma_{h} H_{i}-\mu_{h} H_{r} .
\end{aligned}
$$

All the parameters are non-negative constants for all time $\mathrm{t} \geq 0$. The state variables and parameters for system (2) are described in Table I. By following (Diethelm, 2013), the proposed fractional order dengue model is as follows:

$$
\begin{aligned}
& D^{\alpha} A_{m}=q \phi^{\alpha}\left(1-\frac{A_{m}}{C}\right)-\left(\sigma_{A}{ }^{\alpha}+\mu_{A}{ }^{\alpha}\right) A_{m}, \\
& D^{\alpha} M_{s}=\sigma_{A}^{\alpha} A_{m}-\frac{b^{\alpha} \beta_{m}}{H} M_{s} H_{i}-\mu_{m}{ }^{\alpha} M_{s}, \\
& D^{\alpha} M_{i}=\frac{b^{\alpha} \beta_{m}}{H} M_{s} H_{i}-\mu_{m}{ }^{\alpha} M_{i}, \\
& D^{\alpha} H_{s}=\mu_{h}^{\alpha}\left(H-H_{s}\right)-\frac{b^{\alpha} \beta_{h}}{H} H_{s} M_{i}, \\
& D^{\alpha} H_{i}=\frac{b^{\alpha} \beta_{h}}{H} H_{s} M_{i}-\left(\gamma_{h}{ }^{\alpha}+\mu_{h}{ }^{\alpha}\right) H_{i}, \\
& D^{\alpha} H_{r}=\gamma_{h}{ }^{\alpha} H_{i}-\mu_{h}{ }^{\alpha} H_{r},
\end{aligned}
$$

where, $\alpha \in(0,1]$ is the order of the fractional derivative.

The total human population is given by, $\mathrm{H}=\mathrm{H}_{\mathrm{s}}+\mathrm{H}_{\mathrm{i}}+\mathrm{H}_{\mathrm{r}}$, thus, we can have $\mathrm{H}_{\mathrm{r}}=\mathrm{H}-\mathrm{H}_{\mathrm{s}}+\mathrm{H}_{\mathrm{i}}$. Therefore, system (3) can be reduced to five-dimensional nonlinear system:

$$
\begin{aligned}
& D^{\alpha} A_{m}=q \phi^{\alpha}\left(1-\frac{A_{m}}{C}\right)-\left(\sigma_{A}^{\alpha}+\mu_{A}^{\alpha}\right) A_{m}, \\
& D^{\alpha} M_{s}=\sigma_{A}^{\alpha} A_{m}-\frac{b^{\alpha} \beta_{m}}{H} M_{s} H_{i}-\mu_{m}^{\alpha} M_{s}, \\
& D^{\alpha} M_{i}=\frac{b^{\alpha} \beta_{m}}{H} M_{s} H_{i}-\mu_{m}^{\alpha} M_{i}, \\
& D^{\alpha} H_{s}=\mu_{h}^{\alpha}\left(H-H_{S}\right)-\frac{b^{\alpha} \beta_{h}}{H} H_{S} M_{i}, \\
& D^{\alpha} H_{i}=\frac{b^{\alpha} \beta_{h}}{H} H_{S} M_{i}-\left(\gamma_{h}^{\alpha}+\mu_{h}^{\alpha}\right) H_{i} .
\end{aligned}
$$

\section{STABILITY ANALYSIS}

\section{A. Basic Reproduction Number}

Definition 2 The basic reproduction number denoted by $R_{O}$ is the expected number of secondary infections caused by a single infectious individual during their entire infectious lifetime. 
The expression for the basic reproduction number $\mathrm{R}_{\mathrm{O}}$ is obtained using the next generation matrix approach (van den Driessche \& Watmough, 2002) as follows:

$$
R_{0}=\sqrt{\frac{b^{2 \alpha} \beta_{m} \beta_{h} C\left(q \phi^{\alpha} \sigma_{A}^{\alpha}-\mu_{\mathrm{m}}{ }^{\alpha}\left(\sigma_{\mathrm{A}}{ }^{\alpha}+\mu_{\mathrm{A}}{ }^{\alpha}\right)\right)}{H \mu_{m}^{2 \alpha} q \phi^{\alpha}\left(\gamma_{h}^{\alpha}+\mu_{h}^{\alpha}\right)}} .
$$

\section{B. Equilibrium Points of the Model}

We obtained three equilibrium points for system (4), specifically known as the disease-free equilibrium (DFE) and the positive endemic equilibrium (EE). The trivial DFE is obtained as, $E_{0}=(0,0,0, H, 0)$.Since $A_{m}=0$, the mosquito population is at zero value, thus, no dengue outbreak.

The other DFE that is described as the biologically realistic disease-free equilibrium (BRDFE), is the case when human and vector interact, but no major outbreak occurred.

$$
E_{1}=\left(\bar{A}_{m}, \bar{M}_{s}, 0, H, 0\right),
$$

where $\overline{\mathrm{A}}_{\mathrm{m}}$ and $\overline{\mathrm{M}}_{\mathrm{s}}$ are given by

$$
\overline{\mathrm{A}}_{\mathrm{m}}=\mathrm{C}\left(1-\frac{1}{\mathrm{R}_{\mathrm{m}}}\right), \quad \text { and } \quad \overline{\mathrm{M}}_{\mathrm{s}}=\frac{\sigma_{A}^{\alpha} \overline{\mathrm{A}}_{\mathrm{m}}}{\mu_{m}^{\alpha}},
$$

where $R_{m}=\frac{q \phi^{\alpha} \sigma_{A}^{\alpha}}{\mu_{m}^{\alpha}\left(\sigma_{A}^{\alpha}+\mu_{A}^{\alpha}\right)} \cdot R_{m}$ is defined as the basic number of offspring of the mosquito population.

The positive equilibrium point is called the endemic equilibrium point, denoted by $E_{2}$.

$$
E_{2}=\left(A_{m}^{*}, M_{s}^{*}, M_{i}^{*}, H_{s}^{*}, H_{i}^{*}\right),
$$

where

$$
\begin{aligned}
& A_{m}^{*}=C\left(1-\frac{1}{R_{m}}\right) \\
& M_{s}^{*}=\frac{\sigma_{A}^{\alpha} C\left(1-\frac{1}{R_{m}}\right)\left(1+\mu_{m}^{\alpha}\left(\gamma_{h}^{\alpha}+\mu_{h}^{\alpha}\right) R_{0}^{2}\right)}{\mu_{m}^{\alpha} R_{0}^{2} K_{1}}, \\
& M_{i}^{*}=\frac{\sigma_{A}^{\alpha} C\left(1-\frac{1}{R_{m}}\right)\left(R_{0}^{2}-1\right)}{\mu_{m}^{\alpha} R_{0}^{2} K_{1}}, \\
& H_{s}^{*}=\frac{H K_{2}}{K_{2}+\mu_{m}^{\alpha}\left(\gamma_{h}^{\alpha}+\mu_{h}^{\alpha}\right)\left(R_{0}^{2}-1\right)} \\
& H_{i}^{*}=\frac{H \mu_{m}^{\alpha} \mu_{h}^{\alpha}\left(\left(R_{0}^{2}-1\right)\right.}{K_{2}+\mu_{m}^{\alpha}\left(\gamma_{h}^{\alpha}+\mu_{h}^{\alpha}\right)\left(R_{0}^{2}-1\right)},
\end{aligned}
$$

with $K_{1}=b^{\alpha} \beta_{m} \mu_{h}^{\alpha}+\gamma_{h}^{\alpha}+\mu_{h}^{\alpha}$ and $K_{2}=b^{\alpha} \beta_{m} \mu_{h}^{\alpha}+\mu_{m}^{\alpha}\left(\gamma_{h}^{\alpha}+\mu_{h}^{\alpha}\right)$. Since EE can only be positive values, therefore, $E_{2}$ exists only if $R_{O}>1$. Thus, the following result is established for the existence of equilibrium point.

Theorem 1 (Existence of Equilibrium Points). System (4) always has a disease-free equilibrium point in the absence of the infective population $\left(R_{O}<1\right)$. If $R_{O}>1$, the system of equations (4) has a unique positive endemic equilibrium point.
Theorem 2 (BRDFE stability) The BRDFE of the system of equations (4) is locally asymptotically stable if $R_{O}<1$ and is unstable if $R_{O}>1$.

Proof 1 The disease-free equilibrium is locally asymptotically stable if all the eigenvalues, $\lambda_{i}, i=1,2,3,4,5$ of the Jacobian matrix $J\left(E_{1}\right)$ satisfy the following condition:

$$
\left|\arg \left(\lambda_{i}\right)\right|>\frac{\alpha \pi}{2} .
$$

The Jacobian matrix of the system evaluated at the equilibrium point, $E_{1}$ :

$J\left(E_{1}\right)=\left[\begin{array}{ccccc}-R_{m} k_{1} & 0 & 0 & 0 & 0 \\ \sigma_{A}^{\alpha} & -\mu_{m}^{\alpha} & 0 & 0 & -\frac{b^{\alpha} \beta_{m}}{H} \bar{M}_{s} \\ 0 & 0 & -\mu_{m}^{\alpha} & 0 & \frac{b^{\alpha} \beta_{m}}{H} \bar{M}_{s} \\ 0 & 0 & -b^{\alpha} \beta_{h} & -\mu_{h}^{\alpha} & 0 \\ 0 & 0 & b^{\alpha} \beta_{h} & 0 & -k_{2}\end{array}\right]$, where

$k_{1}=\sigma_{A}^{\alpha}+\mu_{A}^{\alpha}$ and $k_{2}=\gamma_{h}^{\alpha}+\mu_{h}^{\alpha}$. The calculated eigenvalues are $\lambda_{1}=-R_{m}\left(\sigma_{A}^{\alpha}+\mu_{A}^{\alpha}\right), \lambda_{2}=-\mu_{m}^{\alpha}, \lambda_{3}=-\mu_{h}^{\alpha} ;$ the other two roots are determined by the roots of the quadratic equation below:

$$
\lambda^{2}+\left(\mu_{m}^{\alpha}+\gamma_{h}^{\alpha}+\mu_{h}^{\alpha}\right) \lambda+\mu_{m}^{\alpha}\left(\gamma_{h}^{\alpha}+\mu_{h}^{\alpha}\right)\left(1-R_{0}\right)=0 .
$$

Hence, proved that $E_{1}$ is locally asymptotically stable if $R_{O}<1$ and is unstable if $R_{O}>1$ and the condition $R_{m}<1$ is satisfied.

To prove for the global stability of the BRDFE of system (4), we used the Lyapunov function.

Theorem 3 If $R_{0}<1$, then the BRDFE $E_{1}$ of reduced system (4) is globally asymptotically stable in positive invariant set $\Omega$.

Proof 2 We define the Lyapunov function $V_{1}\left(M_{i}, H_{i}\right)$ as follows

$$
V_{1}\left(M_{i}, H_{i}\right)=M_{i}+\frac{\mu_{m}^{\alpha}}{\beta_{h} b^{\alpha}} H_{i} .
$$

The derivative of (7) with respect to $t$ along the solution curves of system (4) is given by

$$
\begin{aligned}
& D^{\alpha} V_{1}(t)=\frac{b^{\alpha} \beta_{m}}{H} M_{S} H_{i}-\mu_{m}^{\alpha} M_{i} \\
& +\frac{\mu_{m}^{\alpha}}{\beta_{h} b^{\alpha}}\left(\frac{b^{\alpha} \beta_{h}}{H} H_{S} M_{i}-\left(\mu_{h}^{\alpha}+\gamma_{h}^{\alpha}\right) H_{i}\right) \\
& =\frac{b^{\alpha} \beta_{m}}{H} M_{S} H_{i}-\mu_{m}^{\alpha} M_{i}+\frac{\mu_{m}^{\alpha}}{H} H_{S} M_{i}-\frac{\left(\mu_{h}^{\alpha}+\gamma_{h}^{\alpha}\right) \mu_{m}^{\alpha}}{\beta_{h} b^{\alpha}} H_{i} \\
& =\left(\frac{b^{\alpha} \beta_{m}}{H} M_{S}-\frac{\mu_{m}^{\alpha}\left(\mu_{h}^{\alpha}+\gamma_{h}^{\alpha}\right)}{\beta_{h} b^{\alpha}}\right) H_{i}-\left(\mu_{m}^{\alpha}-\frac{\mu_{m}^{\alpha}}{H} H_{s}\right) M_{i} \\
& =\left(\frac{b^{\alpha} \beta_{m}}{H} M_{S}-\frac{\mu_{m}^{\alpha}\left(\mu_{h}^{\alpha}+\gamma_{h}^{\alpha}\right)}{\beta_{h} b^{\alpha}}\right) H_{i}-\mu_{m}^{\alpha}\left(1-\frac{H_{s}}{H}\right) M_{i} \quad T h u \\
& \leq\left(\frac{b^{\alpha} \beta_{m}}{H} M_{S}-\frac{\mu_{m}^{\alpha}\left(\mu_{h}^{\alpha}+\gamma_{h}^{\alpha}\right)}{\beta_{h} b^{\alpha}}\right) H_{i} \\
& =\left(\frac{b^{\alpha} \beta_{m}}{H} \frac{\sigma_{A}^{\alpha}}{\mu_{m}^{\alpha}} C\left(1-1 / R_{m}\right)-\frac{\mu_{m}^{\alpha}\left(\mu_{h}^{\alpha}+\gamma_{h}^{\alpha}\right)}{\beta_{h} b^{\alpha}}\right) H_{i} \\
& \text { multiply } 1^{\text {st }} \text { part by } \frac{\beta_{h} b^{\alpha}\left(\mu_{h}^{\alpha}+\gamma_{h}^{\alpha}\right) \mu_{m}^{\alpha}}{\beta_{h} b^{\alpha}\left(\mu_{h}^{\alpha}+\gamma_{h}^{\alpha}\right) \mu_{m}^{\alpha}} \\
& =\frac{\mu_{m}^{\alpha}\left(\mu_{h}^{\alpha}+\gamma_{h}^{\alpha}\right)}{\beta_{h} b^{\alpha}}\left(R_{0}^{2}-1\right) H_{i} .
\end{aligned}
$$

$s$, we established that $D^{\alpha} V_{1}(t)<0$ if $R_{0}<1$ and $V_{1}(t)=0$ if 
and only if $M_{i}=0, H_{i}=0$. Therefore, the largest compact invariant set

$$
\left\{\left(A_{m}, M_{s}, M_{i}, H_{s}, H_{i}\right) \in \Omega: D^{\alpha} V_{1}(t)=0\right\},
$$

is the singleton set $E_{1}$ in $\Omega$. From LaSalle's invariant principle (LaSalle, 1968), every solution that starts in the region $\Omega$ approaches $E_{1}$ as $t \rightarrow \infty$. Hence, the BRDFE $E_{1}$ is globally asymptotically stable for $R_{0}<1$ in $\Omega$.

\section{SENSITIVITY ANALYSIS}

Sensitivity analysis is an essential tool in analysing the importance of each model parameter in disease transmission. It helps us to measure the relative change in a variable when a parameter changes. This is crucial to optimize control measures of the disease. In this study, the sensitivity index is calculated using the normalized sensitivity index.

Definition 3 (Chitnis et al., 2008) The normalised forward sensitivity index of $R_{0}$, that depends differentiably on a parameter $p$, is defined by

$$
\mathrm{Y}_{p}^{R_{0}}=\frac{\partial R_{0}}{\partial p} \times \frac{p}{R_{0}} .
$$

The sensitivity indices revealed the delicacies of variable $R_{0}$ to the model parameters. The positive (negative) index indicate that an increase in the parameter value leads to an increase (decrease) of $R_{0}$ value. The sensitivity index of each parameter in model (4) are depicted in Table 2.

Table 2. Sensitivity indices of $\mathrm{R}_{0}$, for $\alpha=0.9$.

\begin{tabular}{|c|c|c|}
\hline Parameter & $\begin{array}{c}\text { Baseline } \\
\text { values }\end{array}$ & $\begin{array}{c}\text { Sensitivity } \\
\text { indices }\end{array}$ \\
\hline$q$ & 0.8 & -0.0167 \\
\hline$\phi$ & 7.5 & -0.0112 \\
\hline$\sigma_{A}$ & 0.08 & +0.5123 \\
\hline$\mu_{A}$ & 0.25 & +0.0123 \\
\hline$\mu_{m}$ & 0.029 & -1.0167 \\
\hline$\mu_{h}$ & 0.0000365 & -0.00014153 \\
\hline$\beta_{m}$ & 0.375 & +0.5 \\
\hline$\beta_{h}$ & 0.75 & +0.5 \\
\hline$b$ & 0.5 & +0.9 \\
\hline$\gamma_{h}$ & 0.3288 & -0.5232 \\
\hline
\end{tabular}

It follows from Table 2, parameters that related to the death rate of adult mosquitoes, the mosquito biting rate, human recovery rate, and transition rate from the aquatic stage to adult stage mosquito, have highest sensitivity indices towards $R_{0}$. This indicates, for example, an increase in the death rate by $10 \%$ will result in a decrease in the value of $R_{0}$ by $10.17 \%$.

\section{NUMERICAL RESULTS}

Numerical simulation has been performed to validate the stability analysis presented in section 3 . To simulate the results,a MATLAB routine called fde12 established by Garrappa (Garrappa, 2018) is used in this work. The simulations are carried out using the following initial conditions:

$$
\begin{gathered}
H_{s 0}=N_{h}-H_{i 0}, H_{i 0}=2511 \\
A_{m 0}=k N_{h}, M_{s 0}=m N_{h}
\end{gathered}
$$

where $N_{h}=31200000, k=1, m=2$. The final time $t_{\text {end }}=$ 365 days. The initial conditions are chosen based on the real data of dengue cases reported in Malaysia in 2016.
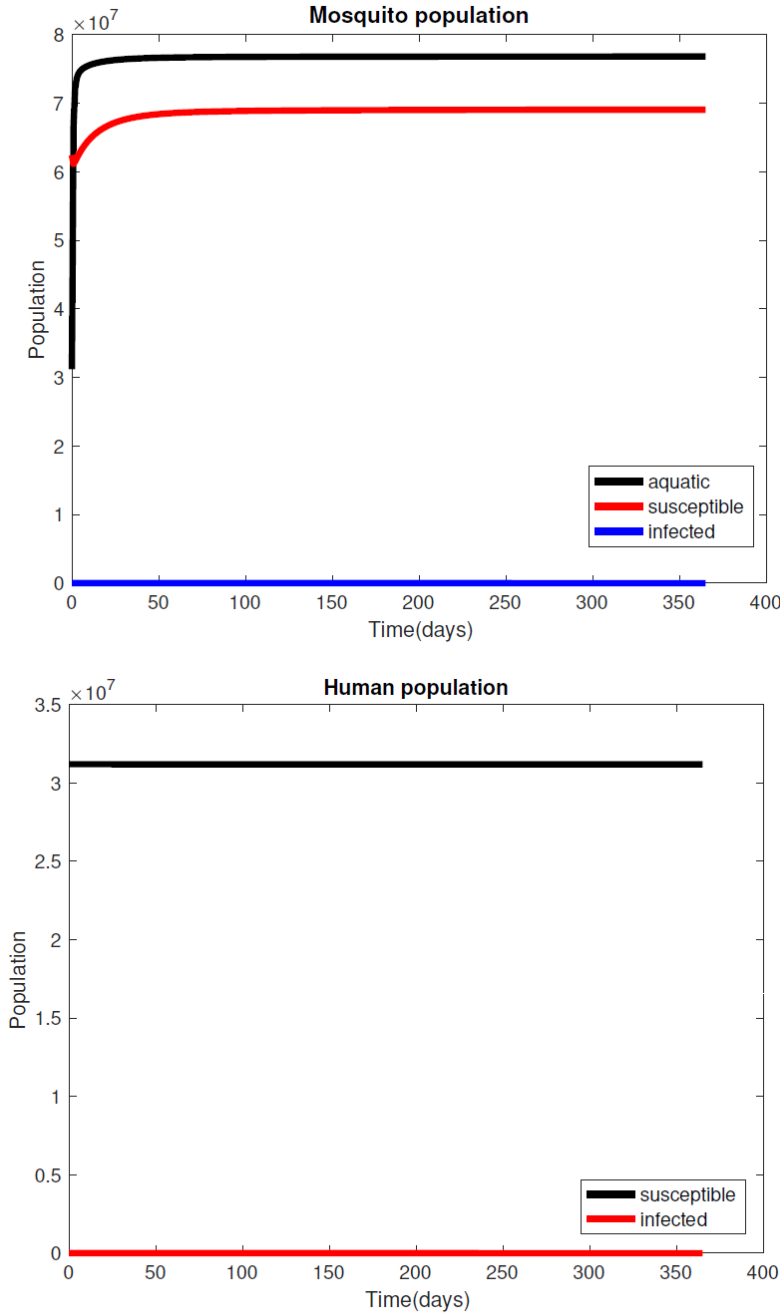

Figure 1.Time series plot for $\alpha=0.9$ and $R_{0}<1$.

Figure 1 shows that all the solution trajectories approach the BRDFE over time when $R_{0}<1$. This confirms the theorem that the BRDFE is globally asymptotically 
stable if $R_{0}<1$

Figure 2 represents the integer order solution as $\alpha=1$. We can observe that for $R_{0}>1$, solutions approach the EE point, both for $\alpha=1$, and also $\alpha=0.9$ in Figure 3. These figures show that $E_{2}$ is a stable EE of system (4). In the case where epidemic occurs $\left(R_{0}>1\right)$, we observed that, if $\alpha=1$ (implies integer order model), the solutions require shorter time to approach the steady state (EE). However, in the fractional order model, when $\alpha=0.9$, more time is needed to reach the EE.
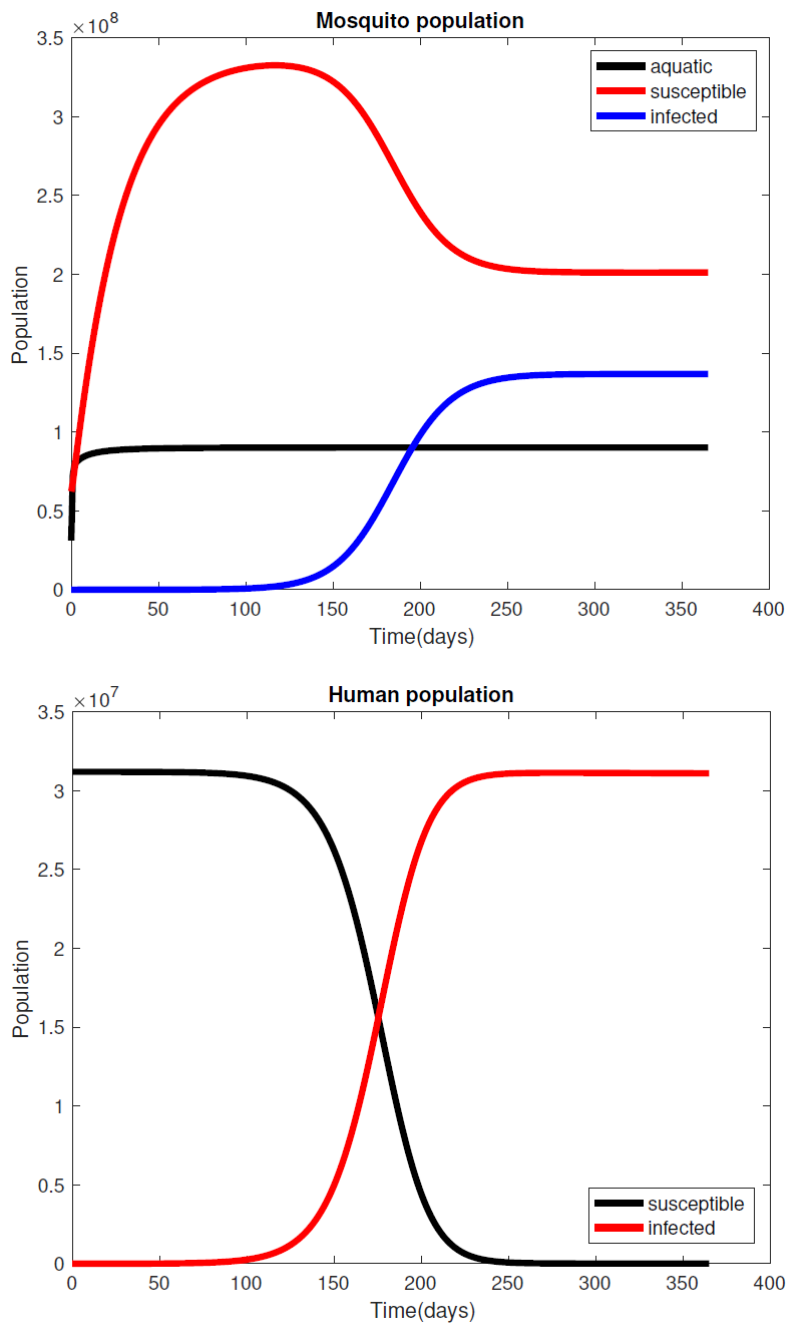

Figure 2. Time series plot for $\alpha=1$ and $R_{0}>1$.

Figure 4 verifies the sensitivity analysis done in section 4. We can see that the infected human population is decreasing as $\mu_{m}$ is increasing, where more time is needed for the major outbreak to be reached. Reversely, when $b$ is increasing, a major epidemic occurs within a short period of time.
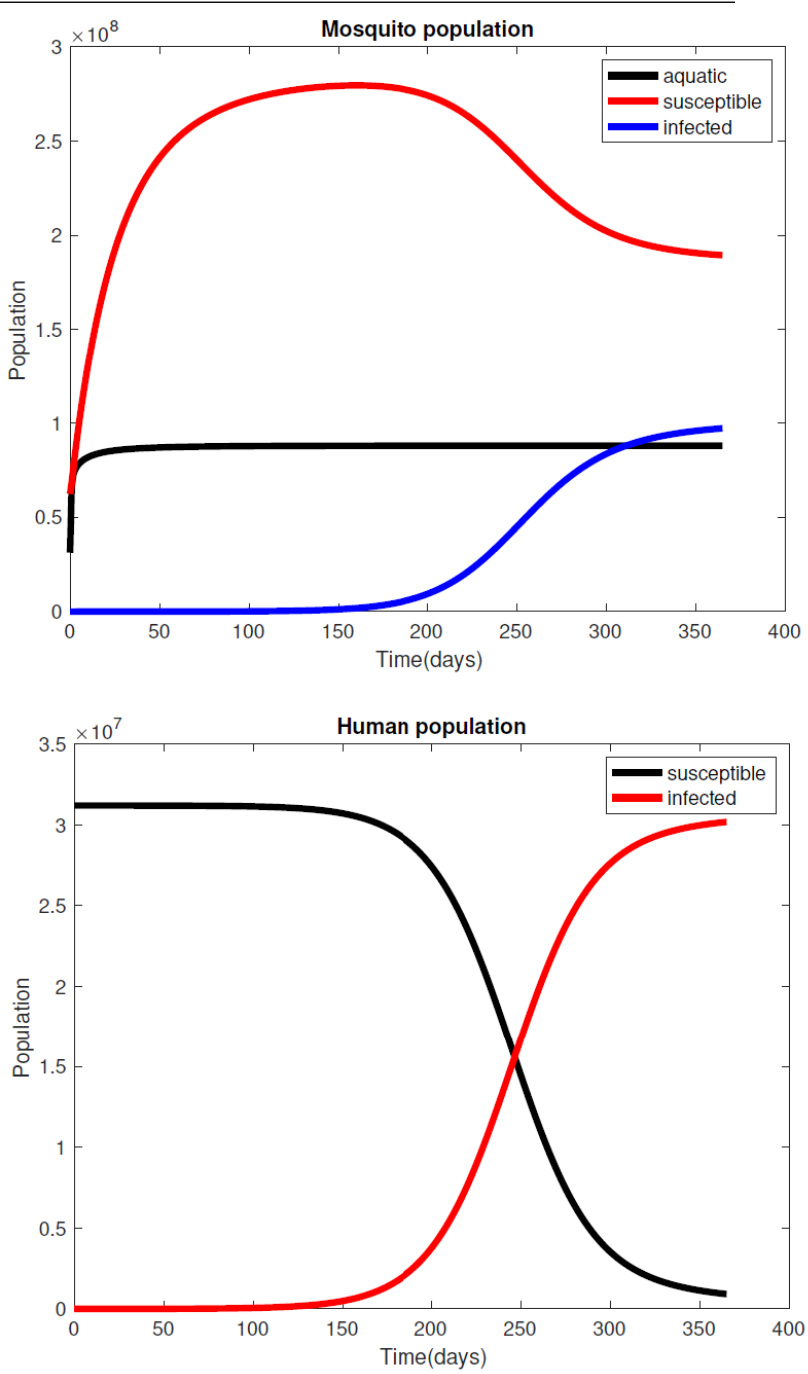

Figure 3.Time series plot for $\alpha=0.9$ and $R_{0}>1$.

\section{CONCLUSION}

Dengue has become a worldwide public health problem. Thus, a well-developed mathematical model is crucial in understanding the dynamics of dengue transmission. In the present study, we have used fractional order model to study the behaviour of the dengue transmission.

This model has shown promising results and provides flexibility to researchers in designing the transmission model by associating memory into the model.

The sensitivity analysis performed shows that any control and prevention measures should target the vector control that can reduce the abundance of immature form and adult female mosquitoes, also reducing mosquitohuman contact rates. 

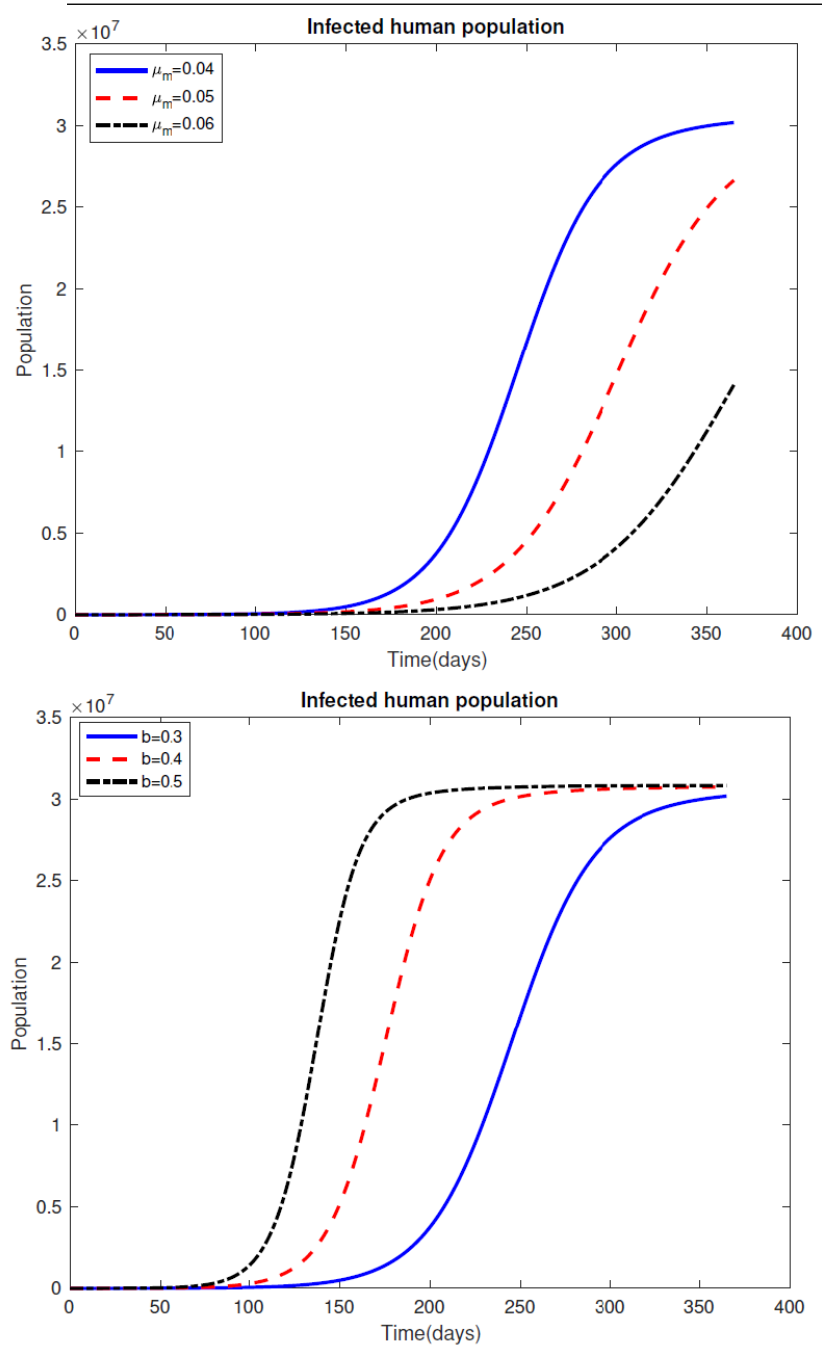

Figure 4.Time series plot for $\alpha=0.9$ and variation in parameter $\mu_{m}$ and $\mathrm{b}$.
VII. ACKNOWLEDGEMENTS

The authors are very grateful for partial financial support by the Universiti Putra Malaysia providing Putra Grant GP-IPS/2018/962500o. The authors also thank the Ministry of Education Malaysia and the Universiti Teknologi Mara.

\section{REFERENCES}

Chitnis, N., Hyman, J.M., Cushing, J.M. 2008, Determining important parameters in the spread of malaria through the sensitivity analysis of a mathematical model. Bulletin of Mathematical Biology, 70(5), 1272-1296.

Derouich, M., Boutayeb, A., Twizell, E.H. 2003, A model of dengue fever. BioMedical Journal in Line Central, 2(1), 1-10.

Diethelm, K. 2013, A fractional calculus based model for the simulation of an outbreak of dengue fever. Nonlinear Dynamics, 71(4), 613-619.

El-Shahed, M., Alsaedi, A. 2011, The fractional SIRC model and Influenza A. Mathematical Problems in Engineering, 2011, 9 pages.
Esteva, L., Vargas, C. 1998, Analysis of a dengue disease transmission model. Mathematical Biosciences, 150(2), 131-151.

Esteva, L. Vargas, C. 1999, A model for dengue disease with variable human population. Journal of Mathematical Biology, 38(3), 220- 240.

Garrappa, R. 2018, Numerical solution of fractional differential equations: a survey and a software tutorial. Mathematics, 6(2).

Hamdan, N. I. and Kilicman, A. 2018, A frac- tional order SIR epidemic model for dengue transmission. Chaos, Solitons and Fractals, 114, 55-62.

Hamdan, N. I. and Kilicman, A. 2019, Analysis of the fractional order dengue transmission model: a case study in Malaysia. Advances in Difference Equations, 
2019.

Hamdan, N. I. and Kilicman, A. 2019, Basic epidemic model of dengue transmission using the fractional order differential equations. Malaysia Journal of Science Special Issue, (1), 1-18.

Kermack, W.O., McKendrick, A.G. 1927, A contribution to the mathematical theory of epidemics. Proceeding of the Royal Society of London, 115(772), 700-721.

LaSalle, J.P. 1968, Stability theory for ordinary differential equations. J. Differ Equations, 41, 57-65.

Petras, I. 2011, Fractional-order nonlinear systems: modeling, analysis and simulation. Higher Education Press, Beijing.

Pinho, S.T.R., Ferreira, C.P., Esteva, L., Barreto, F.R.K., Morato, E., Silva, V.C., Teixeira, M.G.L. 2010, Modelling the dynamics of dengue real epidemics. Phil. Trans. R. Soci., 368(1933), 5679-5693.

Pooseh, S., Rodrigues, H., Torres, D. 2011, Fractional derivatives in dengue epidemics. In: Simos $T$, Psihoyios G, Tsitouras C, Anastassi Z, Numerical analysis and applied mathematics ICNAAM (Melville: American Institute of Physics), 739-742.

Sardar, T., Rana, S., Chattopadhyay, J. 2014, A mathematical model of dengue transmission with memory. Commun Nonlinear SciNumerSimulat, 22, 511-525.

Sardar, T., Rana, S., Bhattacharya, S., Al- Khaled, K., Chattopadhyay, J. 2015, A generic model for a single strain mosquito-transmitted disease with memory on the host and the vector. Mathematical Biosciences, $263,18-36$.

Soewono, E., Supriatna, A.K. 2001, A two- dimensional model for the transmission of dengue fever disease.

Bull. Malaysian Math. Sc. Soc., 24(1), 49-57.

van den Driessche, P., Watmough, J. 2002, Reproduction numbers and sub-threshold endemic equilibria for compartmental models of disease transmission. Mathematical Biosciences, 180(1), 29-48 World Health Organization 2018, Dengue. 